\title{
Divergent roles of tumor suppressor gene Klf6 in ES cell differentiation
}

\author{
Nobuyuki Matsumoto ${ }^{1,2}$, Xiao Zhao ${ }^{1}$, Fumio Itoh $^{2}$ and Scott L. Friedman ${ }^{1}$
}

\begin{abstract}
SUMMARY
Klf6 is a zinc finger transcription factor that has been identified as a tumor suppressor gene inactivated in several human cancers. We previously reported that $\mathrm{Klf}^{-/}$mice are embryonic lethal because of impaired hematopoietic differentiation. In that study, we also found $\mathrm{Klf}^{-/-}$mice have undefinable livers. Combined with this observation and its developmental expression pattern, Klf6 might have a role in in differentiation of non-hematopoietictissues. The aim of this study was to more clearly define the roles of Klf6 in tissue specification using tissue-specific ES cell differentiation systems. We have evaluated three different culture conditions to induce ES cell differentiation, comparing the responses between $\mathrm{Klf6}^{+/+}$and $\mathrm{Klf6}^{-/-}$ES cells. Neuronal differentiation of $\mathrm{Klf6}^{-/-}$ES cells was impaired based on marker gene expression and morphology. Interestingly, $\mathrm{Klf}^{-/-} \mathrm{ES}$ cells began to express Hnf3 $\beta$ mRNA, which identified these cells as endodermal precursors, suggesting that Klf6 ordinarily suppresses endoderm differentiation in this culture condition. Additionally, when $\mathrm{Klf}^{-/}$ES cells were grown in StemPro34 serum free medium, they showed enhanced cardiac differentiation to a greater extent than $\mathrm{Klf}^{+/+}$ES cells, suggesting that Klf6 may have an inhibitory activity in preventing cardiomyogenesis. These findings indicate divergent and tissue- specific roles of Klf6 in ES cell differentiation.
\end{abstract}

Key words: Klf6, ES cell, differentiation.

\section{INTRODUCTION}

Klf6 is a zinc finger transcription factor that has been identified as a tumor suppressor gene inactivated in several human cancers ${ }^{1-3)}$. We previously reported that $\mathrm{Klf6}^{-/}$ mice are embryonic lethal by day 12.5 post fertilization. Their lethality was associated with markedly reduced hematopoiesis and poorly organized yolk sac vascularization. We confirmed this role of Klf6 in differentiation using $\mathrm{Klf6}^{-/-}$embryonic stem (ES) cells, which displayed broad defects in differentiaton ${ }^{4)}$. However, developmental expression of Klf6 is also noted in several neuronal sites, hindgut, heart, lung, kidney and limb buds ${ }^{5)}$. These observations suggested that Klf6 might have broad and divergent roles in tissue differentiation. The aim of this study was to more clearly define the roles of Klf6 in tissue specification, using ES cell differentiation systems that specifically confer tissue specific differentiation.

\section{MATERIALS AND METHODS}

\section{ES cell culture}

ES cells were maintained on irradiated mouse embryonic fibroblast (MEF) feeder cells in Dulbecco's modified essential medium, supplemented with $15 \%$ fetal calf serum (FCS), penicillin, streptomycin, and $2 \%$ leukemia inhibitory factor (LIF) and $1.5 \times 10^{4}$ monothioglycerol (MTG; SigmaAldrich, St Louis, MO). Prior to the initiation of differentiation, ES cells were passaged twice on gelatin-coated dishes in the same media to deplete the population of MEF feeder cells.

\footnotetext{
${ }^{1}$ Division of Liver Diseases, Department of Medicine, Mount Sinai School of Medicine.

${ }^{2}$ Division of Gastroenterology and Hepatology, St. Marianna University School of Medicine. NY 10029, USA. merase Chain Reaction.

Communicated by Hisashi Hirano.

(Received June 16, 2008, Accepted August 12, 2008, Published December 15, 2008)
}

Correspondence address: Scott L. Friedman; Box 1123, Mount Sinai School of Medicine, 1425 Madison Ave, Room 1170F, New York,

Abbreviations: ES cell, embryonic stem cell; EB, embryoid body; FCS, fetal calf serum; IMDM, Iscoves modified Dulbecco medium; LIF, leukemia inhibitory factor; MEF, mouse embryonic fibroblast; MTG, monothioglycerol; RT-PCR, Reverse Transcription-Poly- 


\section{Induction of differentiation}

We examined three different culture conditions to induce ES cell differentiation. The culture protocols are schematically indicated as Fig. 1. We generated embryoid bodies (EBs) in all culture conditions. For the generation of EBs, ES cells were trypsinized into single-cell suspensions and plated at variable densities $(300-4500$ cells $/ \mathrm{ml})$ into each differentiation media.

Culture condition I is for hematopoietic differentiation. ES cells were initially grown in Iscoves modified Dulbecco medium (IMDM) supplemented with 15\% FCS, 5\% Protein Free Medium (PFMHII; Gibco/BRL, NY), $2 \mathrm{mM}$ Lglutamine (Gibco/BRL, NY), $6 \mu \mathrm{l} / \mathrm{ml}$ Transferrin (Roche, Switzerland), 0.5 mM ascorbic acid (Sigma-Aldrich, MO), and $4.5 \times 10^{4} \mathrm{M}$ MTG in 60-mm Petri grade dishes.

In the culture condition II, we induced ES cells to differ-

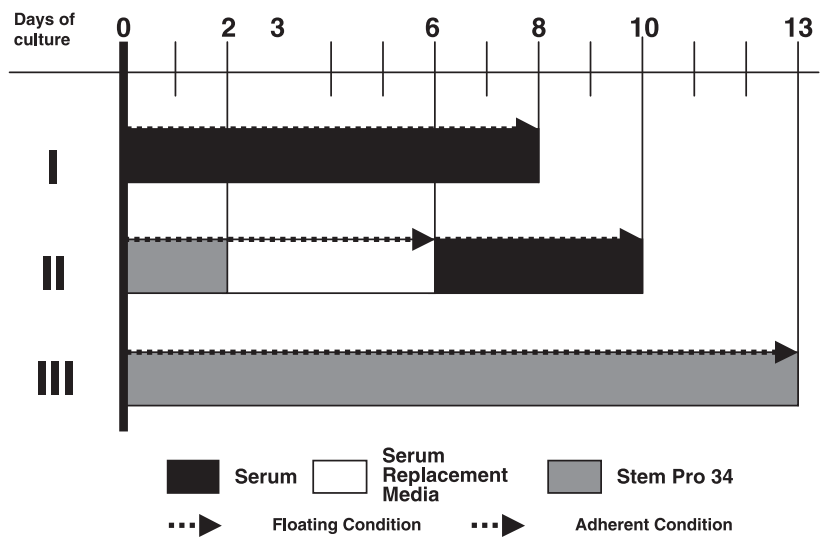

Fig. 1. Schematic diagram of culture conditions used in this study.

ES cells were maintained with LIF contained medium to inhibit spontaneous differentiation until day 0 . ente into a neuronal lineage. EBs were generated in StemPro34 (Gibco/BRL, NY) supplemented with $2 \mathrm{mM}$ Lglutamine, $0.5 \mathrm{mM}$ ascorbic acid, and $4.5 \times 10^{4} \mathrm{M}$ MTG. At day 2 of differentiation, EBs were put into medium, which contains $85 \%$ of IMDM and $15 \%$ of Serum Replacement medium was supplemented with L-glutamine, ascorbic acid and MTG. At day 6, EBs were replated onto a new gelatinized 6 well plates in IMDM supplemented with $15 \%$ FCS, L-glutamine, ascorbic acid, and MTG. This allowed EBs to adhere and differentiate on the culture surface. The appearances and rates of colonies with neurite outgrowth were assessed at day 10. Lastly, we used StemPro34 supplemented with $2 \mathrm{mM}$ L-glutamine, $0.5 \mathrm{mM}$ ascorbic acid, and $4.5 \times 10^{4} \mathrm{M}$ MTG for the culture condition III. We generated EBs in this media, and cultured them for 13 days. At day 13, the percentage of beating EBs was assessed, and EBs were harvested to examine gene expression. All cultures were maintained in a humidified chamber in a $5 \% \mathrm{CO} 2 /$ air mixture at $37^{\circ} \mathrm{C}$.

\section{Reverse Transcription-Polymerase Chain Reaction (RT-PCR)}

Total RNA was extracted from undifferentiated or differentiated ES cells using RNeasy kit (Qiagen, CA), and cDNAs were synthesized from $1 \mu \mathrm{g}$ of total RNA using an RT kit (Promega, WI). Sequences of PCR primers, annealing temperatures, number of cycles, and the sizes of PCR products for each target are indicated in Table 1. Amplified products were visualized by ethidium bromide staining after agarose gel electrophoresis.

\section{RESULTS}

In this study, we utilized three different culture condi-

Table 1. PCR primers and condtions.

\begin{tabular}{|c|c|c|c|c|c|}
\hline Gene Name & Forward & Reverse & Annealing & Cycles & Product size \\
\hline Klf6 & TATCTTCAGGATGAGCCCTGCTAC & AGACTTCACCAATGGGATCAGAGG & 60 & 30 & 276 \\
\hline Rex1 & CGTGTAACATACACCATCCG & GAAATCCTCTTCCAGAATGG & 60 & 35 & 129 \\
\hline E-ras & TTGGGCACAGTGCAAAGATG & GTCATGGTCTTTCACGAAGC & 57 & 35 & 130 \\
\hline Oct4 & AAAGGTGTTCAGCCAGACCA & CAGGGTCTCCGATTTGCATA & 57 & 35 & 151 \\
\hline Fgf5 & GTGTCTCAGGGGATTGTAGGAATACG & GTGAAGGAAAGTTCCGGTTGC & 65 & 35 & 314 \\
\hline Brachyury & CATGTACTCTTTCTTGCTGG & GGTCTCGGGAAAGCAGTGGC & 60 & 35 & 313 \\
\hline Gata1 & CATTGGCCCCTTGTGAGGCCAGAGA & ACCTGATGGAGCTTGAAATAGAGGC & 60 & 35 & 289 \\
\hline Hnf3 $\beta$ & TGGTCACTGGGGACAAGGGAA & GCAACAACAGCAATAGAGAAC & 55 & 35 & 288 \\
\hline $\operatorname{Hnf} 4 \alpha$ & TTCTGCGAACTCCTTCTGGA & AGGAGCAGCACGTCCTTAAA & 55 & 35 & 112 \\
\hline $\operatorname{Ttr}$ & CTCACCACAGATGAGAAG & GGCTGAGTCTCTCAATTC & 55 & 30 & 225 \\
\hline Afp & TCGTATTCCAACAGGAGG & AGGCTTTTGCTTCACCAG & 55 & 30 & 173 \\
\hline Alb & GCTACGGCACAGTGCTTG & CAGGATTGCAGACAGATAGTC & 55 & 35 & 260 \\
\hline Pax6 & GCTTCATCCGAGTCTTCTCCGTTAG & CCATCTTTGCTTGGGAAATCCG & 60 & 40 & 312 \\
\hline Wnt1 & GATTGCGAAGATGAACGCTGTTTC & TCCTCCACGAACCTGTTGACGG & 54 & 40 & 266 \\
\hline NeuroD & ATGACCAAGGCGCGCCTAGA & ACAGGACAGTCACTGTACGCAC & 60 & 35 & 866 \\
\hline Nkx2.5 & CAGTGGAGCTGGACAAAGCC & TAGCGACGGTTCTGGAACCA & 58 & 35 & 216 \\
\hline aMhc & GGAAGAGTGAGCGGCCATCAAGG & CTGCTGGAGGTTATTCCTCG & 58 & 35 & 302 \\
\hline Mef2C & AGCAAGAATACGATGCCATC & GAAGGGGTGGTGGTACGGTC & 58 & 35 & $311,407,431$ \\
\hline$\beta$-actin1 & TGGCATTGTTACCAACTGGG & TGGATGGCTACGTACATGGC & 57 & 25 & 192 \\
\hline
\end{tabular}


tions to induce ES cell differentiation, assessing the impact of KLF6 allelic loss. As previously reported we employed a hematopoietic differentiation system ${ }^{4}$, documenting impaired differentiation in $\mathrm{KLF}^{-/-}$clones (Fig. 2). Instead, there was extended expression of marker genes for the undifferentiated state, including $\operatorname{Rex} 1^{6)}, \mathrm{E}-\mathrm{ras}^{7)}$, and Oct $4^{8)}$, and further differentiation was clearly impaired in $\mathrm{Klf}^{-/-}$ ES cells. In this culture condition, expression of $\mathrm{TTR}^{9)}$, and Pax $6^{10)}$, were also not detected, indicating lack of differentiation into ectoderm and endoderm. Interestingly, the phenotype of $\mathrm{Klf}^{+/-}$ES cells was at the level intermediate between $\mathrm{Klf}^{+/+}$and $\mathrm{Klf}^{-/}$, suggesting a positive and essential effect of Klf6 in hematopoietic differentiation.

Next, we drove ES cells to differentiate into a neuronal lineage using serum free conditions (Fig. 3). At 10 days of culture differentiation, $\mathrm{Klf}^{+/+} \mathrm{ES}$ cells generated EBs with neurite outgrowth. On the other hand, most of $\mathrm{Klf}^{-/-} \mathrm{ES}$ cells failed to generate EBs with neurite outgrowth, and $\mathrm{Klf}^{+/-}$EBs showed an intermediate phenotype between $\mathrm{Klf}^{+/+}$and $\mathrm{Klf6}^{-/-}$(Fig. 3a-d). In contrast, RT-PCR revealed that markers for ectoderm differentiation, such as Pax6, Wnt1 ${ }^{11)}$ and NeuroD ${ }^{12)}$, were expressed even in $\mathrm{Klf}^{-/-}$ EBs. Interestingly, expression of Hnf3 $\beta$ without brachyury was observed in $\mathrm{Klf}^{-/}$EBs, but not in $\mathrm{Klf}^{+/+}$EBs (Fig. 3e), suggesting that Klf6 suppresses endoderm differentiation, but not hematopoietic differentiation in this culture condition.

Finally, in the culture condition III, we cultured ES cells in serum free medium (StemPro34) for 13 days. On day 13, rhythmically beating EBs consistent with a cardiomyocyte phenotype were frequently observed in $\mathrm{Klf}^{-/-} \mathrm{EBs}$. The frequency of beating EBs was about 8 times higher in $\mathrm{Klf}^{-/}$ EBs than in $\mathrm{Klf6}^{+/+}$EBs (Fig. 4a). RT-PCR showed the

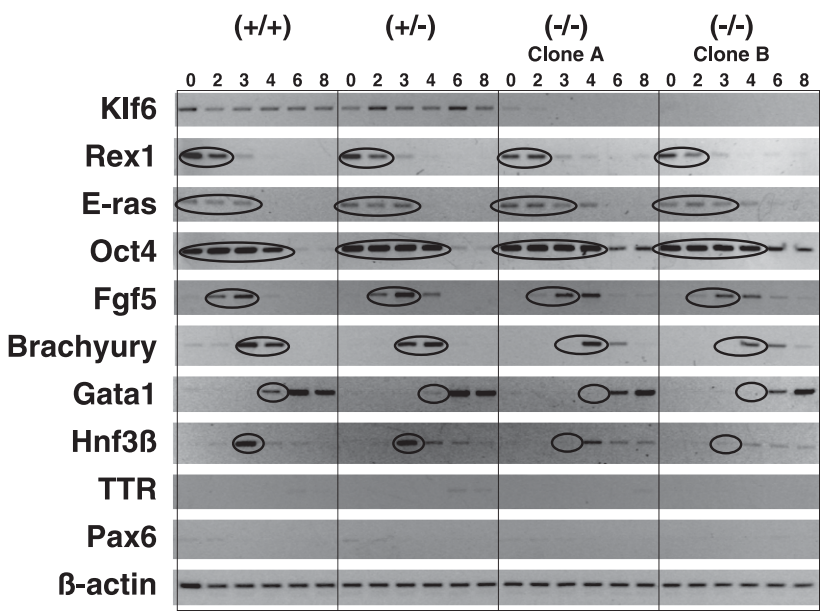

Fig. 2. Involvement of Klf6 in hematopoietic differentiation.

Differentiation of $\mathrm{Klf6}^{-/}$ES cells differentiate slower as previously reported No endoderm and ectoderm marker differentiation are observed, which suggests Klf6 promote hematopoietic differentiation, but it does not suppress endodrem and ectoderm differentiation. stronger expression of $\mathrm{Nkx} 2.5^{13-15)}$ and $\mathrm{aMhc}^{15,16)}$, both of which indicate cardiac differentiation, while Mef2c, which is a critical regulator of myogenesis, and cardiac development ${ }^{15,17)}$, was similarly expressed. We observed not only cardiac markers, but also endoderm and hepatic markers in those EBs. Interestingly, $\mathrm{Klf6}^{-/-}$EBs showed stronger expression of Hnf3 $\beta$, Hnf4a, Afp, and Alb than $\mathrm{Klf}^{+/+}$EBs.

\section{DISCUSSION}

In this study, we have observed divergent effects of Klf6 in tissue specific differentiation depending on the tissue was being generated from ES cells. Specifically, whereas Klf6 is required for hematopoietic and neural differentation, its loss appeared to accelerate cardiomyocyte differentiation, suggesting it ordinarily displays a suppressive effect on this lineage.

In general, $\mathrm{Klf}^{-/-} \mathrm{ES}$ cells differentiate slower than $\mathrm{Klf}^{+/+}$ES cells. Combining the previous observation of ubiquitous expression of $\mathrm{Klf}_{6}{ }^{5,18,19)}$, this transcription factor might be required in the normal differentiation processes of many tissues. However the precise time during tissue specific differentiation at which Klf6 is required is like to differ.

$\mathrm{Klf}^{-/-}$ES cells, cultured in condition I, which induced hematopoietic differentiation, expressed Hnf3 $\beta$, a marker of endoderm precursor cells, on the 3rd day of differentiation, when brachyury positive mesoderm precursor cells arise (Fig. 2), then it is downregulated as cells differentiated into hematopoietic lineage (Fig. 2). Kubo et al. showed Brachyury positive cells can express Hnf3 $\beta$, and these cells can differentiate into endoderm in a culture condition ${ }^{20)}$. These findings support the existence of mesendoderm,
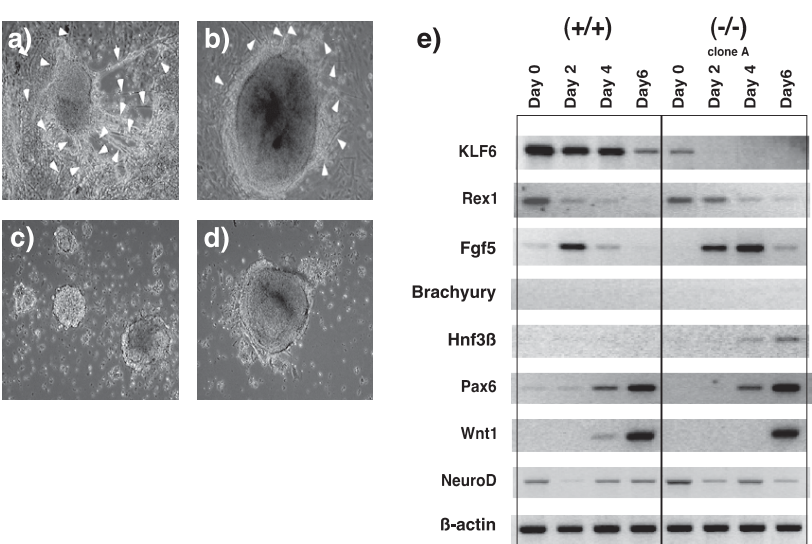

Fig. 3. Klf6 has a critical role in neuronal differentiation.

a) At 10 days of in vitro differentiation, Klf6 ${ }^{+/+}$ES cells could generate EBs with neurite outgrowth. b) $\mathrm{Klf}^{+/-} \mathrm{EBs}$ showed impaired neurite outgrowths. c), d) Klf6 ${ }^{-/-}$ES cells failed to generate EBs with neurite outgrowth. e) RT-PCR revealed that markers for ectoderm differentiation, such as Pax6, Wnt1 and NeuroD, were expressed even in $\mathrm{Klf6}^{-/}$ EBs. Expression of Brachyury was not observed in $\mathrm{Klf}^{+/+}$ and $\mathrm{Klf}^{-/-}$EBs, while expression of Hnf3 $\beta$ was observed in $\mathrm{Klf6}^{-/-}$EBs, but not in Klf6 ${ }^{+/+}$EBs. 

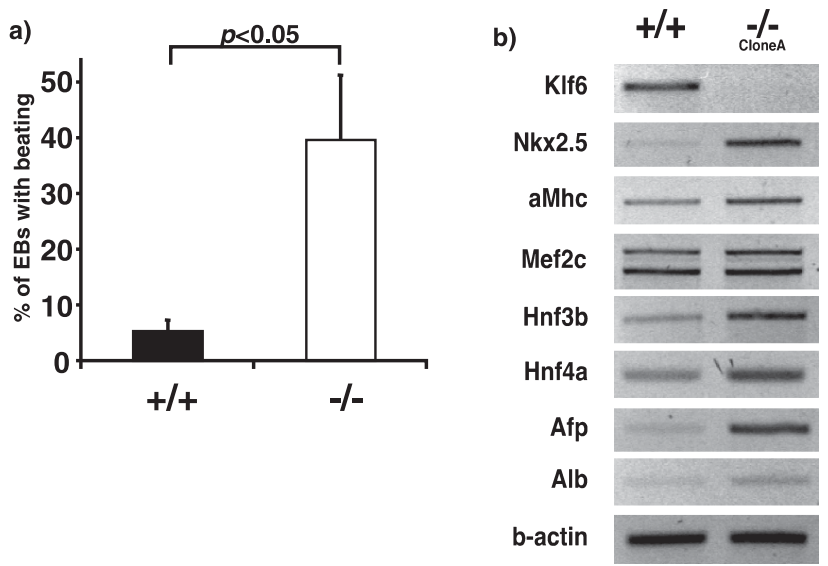

Fig. 4. Negative involvement of Klf6 in myocardial differentiation.

a) Rhythmically beating EBs consistent with a cardiomyocyte phenotype were frequently observed in $\mathrm{Klf}^{-/}$EBs cultured in StemPro34 for 13 days. b) Klf6 ${ }^{-/}$EBs expressed cardiac and hepatic markers stronger than $\mathrm{Klf}^{+/+}$EBs.

which is a common precursor of mesoderm and endoderm. After the mesendoderm stage, with progression of hematopoietic differentiation, further endoderm differentiation is suppressed, which is indicated by the down regulation of Hnf3 $\beta$ and the absence of TTR expression. This phenomenon was similarly observed regardless of Klf6 status, while hematopoietic differentiation was clearly impaired in $\mathrm{Klf6}^{-/-}$EBs. This result suggests that in the culture condition I, the contribution of Klf6 is mainly to guide hematopoietic differentiation, and nothing to do with endoderm differentiation.

When $\mathrm{Klf6}^{--}$ES cells were cultured conditions that promote neuronal differentiation, neurite outgrowths were clearly reduced, although expression of neuronal marker genes were similar. These findings suggest that Klf6 may primarily involve morphological development of neuronal tissues. Interestingly, $\mathrm{Klf6}^{-/-}$ES cells expressed HNF3 $\beta$ in this condition, suggesting one of the roles of Klf6 in neuronal differentiation may be suppression of other lineage differentiation including hepatic differentiation. On the other hand, no expression of brachyury in $\mathrm{Klf}^{+/+}$and $\mathrm{Klf}^{-/-}$ EBs suggests Klf6 does not suppress mesoderm differentiation in the condition II.

$\mathrm{Klf6}^{-/-}$ES cells differentiated into cardiac muscle cells more than $\mathrm{Klf}^{+/+}$ES cells, which, as noted above, suggesting that Klf6 exerts a suppressive effect on myocardial differentiation in the condition III. The number of beating EBs was significantly increased in $\mathrm{Klff}^{-/-}$ES cells, and expression of $\mathrm{Nkx} 2.5$ and aMhc were stronger than in $\mathrm{Klff}^{+/+}$EBs. However, further refinements in specific culture conditions are required to definitively address the role of Klf6 in myocardial differentiation.

Collectively, these results indicate that Klf6 is involved in tissue specification through induction and/or suppression of lineage differentiation. While underlying mechanisms are unclear, an additional element worth considering is the contribution of Klf6 splice forms to their activity during development. Klf6 is the only Kruppel-like factor, in which splicing variants are reported. These alternative transcript products suppress the full length Klf6 function by competitive inhibition ${ }^{21,22)}$. While splice forms have not yet been uncovered in a development system, this question merits further analysis.

In conclusion, these findings indicate divergent and tissue- specific roles of Klf6 in ES cell differentiation. The promotive and suppressive effects of Klf6 in lineage-specific differentiations are clearly complex and are likely to depend on both the temporal and spatial context of the specific micro-environment.

\section{REFERENCES}

1) Kremer-Tal S, Reeves HL, Narla G, Thung SN, Schwartz M, Difeo A, Katz A, Bruix J, Bioulac-Sage P, Martignetti JA, Friedman SL. Frequent inactivation of the tumor suppressor Kruppel-like factor 6 (KLF6) in hepatocellular carcinoma. Hepatology 2004;40:1047-1052.

2) Narla G, Heath KE, Reeves HL, Li D, Giono LE, Kimmelman AC, Glucksman MJ, Narla J, Eng FJ, Chan AM, Ferrari AC, Martignetti JA, Friedman SL. KLF6, a candidate tumor suppressor gene mutated in prostate cancer. Science 2001;294:2563-2566.

3) Reeves HL, Narla G, Ogunbiyi O, Haq AI, Katz A, Benzeno S, Hod E, Harpaz N, Goldberg S, Tal-Kremer S, Eng FJ, Arthur MJ, Martignetti JA, Friedman SL. Kruppel-like factor 6 (KLF6) is a tumor-suppressor gene frequently inactivated in colorectal cancer. Gastroenterology 2004; 126:1090-1103.

4) Matsumoto N, Kubo A, Liu H, Akita K, Laub F, Ramirez F, Keller G, Friedman SL. Developmental regulation of yolk sac hematopoiesis by Kruppel-like factor 6. Blood 2006;107:1357-1365.

5) Laub F, Aldabe R, Ramirez F, Friedman S. Embryonic expression of Kruppel-like factor 6 in neural and nonneural tissues. Mech Dev 2001;106:167-170.

6) Mongan NP, Martin KM, Gudas LJ. The putative human stem cell marker, Rex-1 (Zfp42): structural classification and expression in normal human epithelial and carcinoma cell cultures. Mol Carcinog 2006;45:887-900.

7) Takahashi K, Mitsui K, Yamanaka S. Role of ERas in promoting tumour-like properties in mouse embryonic stem cells. Nature 2003;423:541-545.

8) Chambers I, Smith A. Self-renewal of teratocarcinoma and embryonic stem cells. Oncogene 2004;23:7150-7160.

9) Abe K, Niwa H, Iwase K, Takiguchi M, Mori M, Abe SI, Abe K, Yamamura KI. Endoderm-specific gene expression in embryonic stem cells differentiated to embryoid bodies. Exp Cell Res 1996;229:27-34.

10) Roche E, Sepulcre P, Reig JA, Santana A, Soria B. Ectodermal commitment of insulin-producing cells derived from mouse embryonic stem cells. Faseb J 2005; 19:1341-1343.

11) Dickinson ME, Krumlauf R, McMahon AP. Evidence for a 
mitogenic effect of Wnt-1 in the developing mammalian central nervous system. Development 1994;120:14531471.

12) Lee JE, Hollenberg SM, Snider L, Turner DL, Lipnick N, Weintraub H. Conversion of Xenopus ectoderm into neurons by NeuroD, a basic helix-loop-helix protein. Science 1995;268:836-844.

13) Tanaka M, Chen $Z$, Bartunkova S, Yamasaki N, Izumo S. The cardiac homeobox gene Csx/Nkx2.5 lies genetically upstream of multiple genes essential for heart development. Development 1999;126:1269-1280.

14) Jamali M, Rogerson PJ, Wilton S, Skerjanc IS. Nkx2-5 activity is essential for cardiomyogenesis. J Biol Chem 2001;276:42252-42258.

15) Makino S, Fukuda K, Miyoshi S, Konishi F, Kodama H, Pan J, Sano M, Takahashi T, Hori S, Abe H, Hata J, Umezawa A, Ogawa S. Cardiomyocytes can be generated from marrow stromal cells in vitro. J Clin Invest 1999; 103:697-705.

16) Wobus AM, Kaomei G, Shan J, Wellner MC, Rohwedel J, Ji G, Fleischmann B, Katus HA, Hescheler J, Franz WM. Retinoic acid accelerates embryonic stem cell-derived cardiac differentiation and enhances development of ventricular cardiomyocytes. J Mol Cell Cardiol 1997;29: 1525-1539.

17) Lin Q, Schwarz J, Bucana C, Olson EN. Control of mouse cardiac morphogenesis and myogenesis by transcription factor MEF2C. Science 1997;276:1404-1407.

18) Fischer EA, Verpont MC, Garrett-Sinha LA, Ronco PM, Rossert JA. Klf6 is a zinc finger protein expressed in a cell-specific manner during kidney development. J Am Soc Nephrol 2001;12:726-735.

19) Nakamura H, Chiambaretta F, Sugar J, Sapin V, Yue BY. Developmentally regulated expression of KLF6 in the mouse cornea and lens. Invest Ophthalmol Vis Sci 2004;45:4327-4332.

20) Kubo A, Shinozaki K, Shannon JM, Kouskoff V, Kennedy M, Woo S, Fehling HJ, Keller G. Development of definitive endoderm from embryonic stem cells in culture. Development 2004;131:1651-1662.

21) Narla G, DiFeo A, Yao S, Banno A, Hod E, Reeves HL, Qiao RF, Camacho-Vanegas O, Levine A, Kirschenbaum A, Chan AM, Friedman SL, Martignetti JA. Targeted inhibition of the KLF6 splice variant, KLF6 SV1, suppresses prostate cancer cell growth and spread. Cancer Res 2005;65:5761-5768.

22) Camacho-Vanegas O, Narla G, Teixeira MS, DiFeo A, Misra A, Singh G, Chan AM, Friedman SL, Feuerstein BG, Martignetti JA. Functional inactivation of the KLF6 tumor suppressor gene by loss of heterozygosity and increased alternative splicing in glioblastoma. Int J Cancer 2007;121:1390-1395. 